\title{
The year in review: recent advances in musculoskeletal radiology and biology
}

\author{
Ravi S. Kamath $\cdot$ Hugue A. Ouellette
}

Published online: 10 November 2009

(C) ISS 2009

The year 2009 has witnessed many scientific advances pertinent to the musculoskeletal system. The medical literature is so vast that substantial effort is required to keep abreast of new developments, even in this one field. Relevant papers may be found in a variety of journals, including those focused on our subspecialty, those on general radiology or surgery, or those on all branches of science and medicine (Table 1).

We have undertaken a review to summarize interesting articles related to musculoskeletal radiology and biology that have appeared this year. We scoured the 27 journals named in Table 1 to identify the most exciting or potentially influential papers published during the calendar year 2009 that could affect our current or future clinical practice. As with any such review, our list is highly subjective and is certainly incomplete, but we hope that it will serve as a stimulus for discussion and further exploration.

For the purposes of this review, we have grouped papers into five broad categories:

1. Those that increase our understanding of the basic biology of physiological or disease processes

2. Clinical trials that demonstrate the efficacy of disease treatments

3. Studies that examine practical use of common radiological examinations

4. Papers that present new uses for existing or emerging technologies

5. Those that present new methods for carrying out interventional procedures

R. S. Kamath $(\varangle) \cdot$ H. A. Ouellette

Division of Musculoskeletal Imaging and Intervention,

Department of Radiology, Massachusetts General Hospital,

Boston, MA 02114, USA

e-mail: rkamath@partners.org

\section{Basic science}

We selected three papers that significantly increased our understanding of skeletal biology. One [1] reports a key link between the regulation of bone mass and metabolism via brain-derived serotonin (BDS), which stimulates bone formation and appetite by binding to neurons and antagonizing the function of leptin, a fat cell-derived hormone that is an important regulator of bone turnover and energy expenditure. Patients who take selective serotonin reuptake inhibitors (SSRIs) for psychiatric disorders are at increased risk of osteoporotic fractures; such medications may cause osteoporosis by shifting the balance between BDS and leptin. By increasing understanding of the hormonal regulation of bone mass, this paper represents an important step toward developing therapies for metabolic disorders, such as obesity, diabetes, and anorexia, that do not compromise bone mass.

The other two papers reveal important features about the biology of bone metastases. The first [2] identifies a mechanism by which two tumor-produced metalloproteinases release epidermal growth factor (EGF)-like ligands to activate osteoclastogenesis, thereby disrupting normal bone remodeling and promoting osteolysis. EGF receptor (EGFR) inhibitors block osteolytic bone metastases, and therefore they may have therapeutic benefits even in patients whose primary tumors are nonresponsive to EGFR inhibitors. Furthermore, the metalloproteinases themselves represent novel targets for preventing bone metastases. The second paper [3] identifies key components of tumor-nerve interactions that are responsible for bone pain from metastatic carcinomas and sarcomas. The authors discovered that receptors for granulocyte- and granulocyte-macrophage colony stimulating factors (G- and GM-CSF) are expressed on sensory nerves and that these 
Table 1 List of journals surveyed

Acta Radiologica
American Journal of Roentgenology
Arthroscopy
Bone
Cell
Clinical Orthopedics
Current Biology
Emergency Radiology
European Journal of Radiology
European Radiology
Genes and Development
Investigative Radiology
JAMA
J Bone Mineral Research
J Bone Joint Surgery UK
J Bone Joint Surgery USA
J Computer Assisted Tomography
J Magnetic Resonance Imaging
Lancet
Magnetic Resonance Imaging
Nature
Nature Medicine
New England Journal of Medicine
Osteoporosis International
Radiology
Science
Skeletal Radiology

factors sensitize nerves and promote growth of nerve endings. Interruption of G- and GM-CSF signaling reduces tumor growth and nerve remodeling and decreases bone cancer pain. These receptors represent potential therapeutic targets for managing painful skeletal metastases.

\section{Clinical trials}

We selected four clinical trials that evaluated treatments for musculoskeletal disorders. Two randomized trials examining the use of vertebroplasty for treatment of painful osteoporotic compression fractures received extensive coverage in the lay press. The first [4] identified patients with unhealed fractures less than 12 months old on MRI. The 71 patients who completed the study were randomly assigned to undergo either vertebroplasty or a sham procedure. Patients who underwent vertebroplasty did not report significantly improved pain, physical function, or quality of life at 1 week or 1,3 , or 6 months compared with control patients. Results were consistent irrespective of symptom duration, sex, treatment center, or presence of other fractures $(P>0.10$ for all). The second [5] randomly assigned 131 patients to receive either vertebroplasty or a sham procedure. For the primary outcomes, scores on a disability questionnaire and pain ratings at 1 month, no significant difference was observed between the two groups $(P=0.49$ and 0.19 respectively). Although the difference was not significant, the authors did note a "trend" toward a higher rate of clinically meaningful pain improvement in the vertebroplasty group ( $64 \%$ vs $48 \%, P=0.06)$. However, this study was weakened by allowing crossover between the two groups at 1 month, which limited the ability to interpret differences in outcomes after this time. Since an estimated 750,000 Americans have vertebral compression fractures, roughly $\$ 12-18$ billion/year is spent on their care, and the number of vertebroplasties performed during the past 6 years has doubled, these studies may significantly alter the way in which we manage such patients.

Two other papers presented results of trials evaluating therapies for common inflammatory arthropathies. The first [6] studied patients with early rheumatoid arthritis (RA) refractory to methotrexate (MTX) therapy and compared the addition of conventional disease-modifying antirheumatic drugs (DMARDs, sulfasalazine and hydroxychloroquine) versus the addition of a tumor necrosis factor (TNF) antagonist (infliximab). The 258 patients with RA refractory to MTX were randomly assigned to receive either a DMARD or infliximab. Infliximab was found to be superior to DMARDs, with good clinical response in $39 \%$ and $25 \%$ of patients respectively (RR 1.59, $P=0.0160$ ). However, whether infliximab becomes a mainstay of treatment remains to be seen, since TNF antagonists are considerably more expensive than conventional antirheumatic drugs. Another paper [7] reported results of a phase II randomized trial for an interleukin-12/23 antagonist (ustekinumab) to treat psoriatic arthritis. The 124 participants were randomly assigned to receive either ustekinumab or placebo; after 12 weeks, the two groups crossed over. Patients were followed for 36 weeks, with the primary outcome being clinical response as measured by ACR20 at week 12. By these criteria, ustekinumab was found to significantly reduce signs and symptoms of psoriatic arthritis, with $42 \%$ of patients achieving the primary endpoint versus $14 \%$ of control patients $(P=0.0002)$. For those with significant skin involvement, $52 \%$ receiving ustekinumab had a $75 \%$ or greater improvement in the psoriasis area and in the severity index score versus $5 \%$ for the control group $(P<0.0001)$. This study demonstrated that ustekinumab is effective and safe for treating active psoriatic arthritis and substantiated the role of interleukin $12 / 23$ in psoriatic arthritis, a role that was previously only well-established in animal studies. 


\section{Clinical radiology}

We selected two papers that studied the practical use of radiological examinations. The first [8] was a meta-analysis that compared the effects of routine, immediate imaging of the lumbar spine (radiography, MRI, or CT) versus usual clinical care without imaging for patients without historical or clinical features of a serious condition (fracture, cancer, infection, or cauda equina syndrome). Six trials were included, with a total of 1,804 randomized patients. The authors found no significant difference between immediate imaging and usual care without imaging at both short term ( $<3$ months) and long term (6-12 months) followup in terms of pain and function, quality of life, mental health, and overall improvement. Pooled estimates were close to zero and, in some cases, slightly favored the non-imaging strategy. Although this study suffers from limitations inherent in any meta-analysis (particularly heterogeneity of the included trials), the results support the notion that imaging for low back pain should be reserved for patients in whom a serious underlying condition is suspected.

The second paper [9] examined inter-observer variability among 11 general radiologists with varying levels of experience. Radiologists were asked to evaluate the same 52 knee MRI examinations for anterior cruciate ligament (ACL), meniscal, and cartilage pathology, and their reports were compared with intra-operative findings. MRI was acquired on $1.0 \mathrm{~T}, 1.5 \mathrm{~T}$, and $3.0 \mathrm{~T}$ systems, so the relationship between field strength and accuracy was also assessed. Overall, the authors noted significant interobserver variability, with correlation values ranging from 0.370 for cartilage lesions to 0.597 for meniscal tears. Correlation did not increase with radiologist experience or field strength. The rate of false positives was highly observer-dependent and was significantly higher among less experienced radiologists, but was not affected by field strength. Thus, the authors concluded that radiologist experience is more important than field strength for determining accuracy of interpretation.

\section{Imaging applications}

We selected three papers that examined the use of existing or emerging imaging technologies for new clinical applications. The first [10] studied the use of diffusion tensor imaging (DTI) in patients with carpal tunnel syndrome (CTS). The authors found that fractional anisotropy, radial diffusivity, and apparent diffusion coefficient differed significantly between healthy subjects and patients with CTS $(P<0.0002)$. In most cases, diagnosis of CT is straightforward; however, for patients with inconclusive clinical findings, this study presents a new application for DTI in diagnosing CTS.

The second paper [11] examined the utility of another existing MRI technology, diffusion-weighted imaging (DWI), for detecting skeletal metastases. The 115 patients in the study with non-small cell lung cancer prospectively underwent bone scintigraphy, PET/CT, and whole-body MRI before treatment. Specificity and accuracy of wholebody MRI with DWI was found to be significantly higher than those of bone scintigraphy and PET/CT $(P<0.05)$. Moreover, whole-body MRI with DWI was found to have improved sensitivity and accuracy compared with MRI alone $(P<0.05)$. Although this is not the first paper to study the use of DWI for evaluating metastases, it provides strong evidence supporting the utility of employing MRI and DWI for oncological staging.

The third paper [12] examines the use of a new technique, high-resolution isotropic 3D-sodium MRI to evaluate osteoarthritis (OA) of the knee. Loss of proteoglycan (PG) is a first step in the cascade that leads to OA; because PG content is proportional to $\mathrm{Na}$ content, sodium concentration reflects the integrity of the cartilage. Because in vivo ${ }^{23} \mathrm{Na}$ NMR signal is $>20,000 \mathrm{x}$ weaker than that of ${ }^{1} \mathrm{H}$, prior studies of Na MRI have suffered from poor signal-to-noise ratios or excessively long scan times. To surmount these limitations, the authors used ultrahigh field strength $(7 \mathrm{~T})$ with $\mathrm{Na}$ MRI for the first time, demonstrating the feasibility of acquiring high-resolution images of the whole knee joint in less than 15 min using a 3D-radial acquisition and an ultrashort echo time. These results point to the possible clinical application of this technology for characterizing OA in the near future.

\section{Intervention}

We selected one paper that examined a new method for carrying out interventional procedures. This paper [13] reported the use of an electromagnetic-based tracking and navigation system for real-time monitoring of needle position during spine intervention. For this technique, the needle has a small coil integrated into the tip; an electromagnetic field induces voltage in the coil dependent on position and orientation, and this voltage is measured by a navigation system. This system was used to access lumbar facet joints and intervertebral discs in a spine phantom, pig cadavers, and anesthetized pigs. Pre-procedure CTs were used to plan trajectories, and needles were passed under real-time monitoring. For facet joint puncture, mean needle positioning errors were $0.4 \mathrm{~mm}$ in the phantom, $2.8 \mathrm{~mm}$ ex vivo, and $3.0 \mathrm{~mm}$ in vivo with a mean needle track length of $>50 \mathrm{~mm}$. Intervertebral discs were successfully punctured on the first attempt in $15 / 15$ tries in the phantom, 
$12 / 15$ ex vivo, and 14/15 in vivo. These results demonstrate the promise of this type of system for clinical use in interventional radiology, which may improve accuracy and safety and decrease radiation dose for CT-guided procedures.

\section{Summary}

Here, we have presented brief summaries of 13 interesting papers published in 2009 that pertain to the musculoskeletal system. Some of these discoveries may see clinical use within the next few years, whereas others represent early basic discoveries that are far removed from direct clinical application. We hope that the breadth of research summarized here conveys at least in part the extent of relevant advances made during the past year, and we look forward to reading papers in coming years that build on these results.

\section{References}

1. Yadav VK, Oury F, Suda N, Liu ZW, Gao XB, Confavreux C. A serotonin-dependent mechanism explains the leptin regulation of bone mass, appetite, and energy expenditure. Cell. 2009;138 (5):976-89.

2. Lu X, Wang Q, Hu G, Van Poznak C, Fleisher M, Reiss M. ADAMTS1 and MMP1 proteolytically engage EGF-like ligands in an osteolytic signaling cascade for bone metastasis. Genes Dev. 2009;23(16):1882-94.

3. Schweizerhof M, Stösser S, Kurejova M, Njoo C, Gangadharan V, Agarwal N. Hematopoietic colony-stimulating factors mediate tumor-nerve interactions and bone cancer pain. Nat Med. 2009;15 (7):802-7.

4. Buchbinder R, Osborne RH, Ebeling PR, Wark JD, Mitchell P, Wriedt C. A randomized trial of vertebroplasty for painful osteoporotic vertebral fractures. N Engl J Med. 2009;361 (6):557-68.

5. Kallmes DF, Comstock BA, Heagerty PJ, Turner JA, Wilson DJ, Diamond TH. A randomized trial of vertebroplasty for osteoporotic spinal fractures. N Engl J Med. 2009;361(6):569-79.

6. Van Vollenhoven RF, Ernestam S, Geborek P, Petersson IF, Cöster $\mathrm{L}$, Waltbrand $\mathrm{E}$. Addition of infliximab compared with addition of sulfasalazine and hydroxychloroquine to methotrexate in patients with early rheumatoid arthritis (Swefot trial): 1-year results of a randomised trial. Lancet. 2009;374(9688):459-66.

7. Gottlieb A, Menter A, Mendelsohn A, Shen YK, Li S, Guzzo C. Ustekinumab, a human interleukin 12/23 monoclonal antibody, for psoriatic arthritis: randomised, double-blind, placebocontrolled, crossover trial. Lancet. 2009;373(9664):633-40.

8. Chou R, Fu R, Carrino JA, Deyo RA. Imaging strategies for lowback pain: systematic review and meta-analysis. Lancet. 2009;373 (9662):463-72.

9. Krampla W, Roesel M, Svoboda K, Nachbagauer A, Gschwantler M, Hruby W. MRI of the knee: how do field strength and radiologist's experience influence diagnostic accuracy and interobserver correlation in assessing chondral and meniscal lesions and the integrity of the anterior cruciate ligament? Eur Radiol. 2009;19(6):1519-28.

10. Stein D, Neufeld A, Pasternak O, Graif M, Patish H, Schwimmer E. Diffusion tensor imaging of the median nerve in healthy and carpal tunnel syndrome subjects. J Magn Reson Imaging. 2009;29 (3):657-62.

11. Takenaka D, Ohno Y, Matsumoto K, Aoyama N, Onishi Y, Koyama H. Detection of bone metastases in non-small cell lung cancer patients: comparison of whole-body diffusion-weighted imaging (DWI), whole-body MR imaging without and with DWI, whole-body FDG-PET/CT, and bone scintigraphy. J Magn Reson Imaging. 2009;30(2):298-308.

12. Wang L, Wu Y, Chang G, Oesingmann N, Schweitzer ME, Jerschow A. Rapid isotropic 3D-sodium MRI of the knee joint in vivo at 7 T. J Magn Reson Imaging. 2009;30(3):606-14.

13. Bruners P, Penzkofer T, Nagel M, Elfring R, Gronloh N, SchmitzRode T. Electromagnetic tracking for CT-guided spine interventions: phantom, ex-vivo and in-vivo results. Eur Radiol. 2009;19 (4):990-4. 